\title{
Nonlinear Dynamic Transformer Time-Domain Identification for Power Converter Applications
}

\author{
Tue T. Vu, Student Member, IEEE, Seamus O'Driscoll, and John V. Ringwood, Senior Member, IEEE
}

\begin{abstract}
For flyback converter applications, an accurate model of the transformer is necessary for simulation studies, as well as a basis for model-based controller design. In general, transformer modeling has either focused on the winding model, using frequency-domain methods, or on the nonlinear core model, using time-domain methods. Nonlinear modeling is confined to the time domain and certain difficulties have precluded the use of timedomain methods for winding model estimation, resulting in the lack of integrated modeling approaches. This paper focuses on identifying a complete nonlinear dynamic model of a 3-winding transformer using time-domain system identification approaches. Our study demonstrates a possible way to handle the difficulties of working in the time domain and provides a model at least as accurate as that obtained with the frequency response data. In addition to the parameters of the Jiles-Atherton model, which is used to describe the nonlinear core behavior, the air-gap length is also computed from the experimental data to enhance the core model accuracy. The obtained transformer winding model, core model, and full model are experimentally verified.
\end{abstract}

Index Terms-Magnetic hysteresis, modeling, system identification, time-domain analysis, transformer cores, transformer windings.

\section{INTRODUCTION}

G IVEN the success of the primary-side regulation (PSR) for flyback converters in discontinuous conduction mode (DCM) [1], the extension to continuous conduction mode $(\mathrm{CCM})$, with the aim of achieving a higher performance and a lower production cost, is of significant interest. However, the study of PSR in CCM requires an accurate model of the flyback transformer, particularly at high frequencies.

The topic of modeling a high-frequency transformer has been investigated previously [2]-[15]. In general, most studies focus either on extracting a frequency-dependent winding model with a linear core assumption [3]-[7], or on modeling nonlinear properties of a specific magnetic material using a highamplitude and high-frequency excitation voltage [8]-[12]. Although it is claimed in [14] and [15] that they are able to handle the frequency-dependent and hysteresis effects inside the

Manuscript received October 19, 2012; revised February 6, 2013; accepted February 25, 2013. Date of current version July 18, 2013. This work was supported by the Semiconductor Research Corporation, Dallas, under Contract 2008-HC-1836. Recommended for publication by Associate Editor B. Ferreira.

T. T. Vu and J. V. Ringwood are with the Department of Electronic Engineering, National University of Ireland, Maynooth, Kildare 00353, Ireland (e-mail: ttrongvu@eeng.nuim.ie; john.ringwood@eeng.nuim.ie).

S. O'Driscoll is with Texas Instruments, Cork, Ireland (e-mail: seamusodriscoll@ti.com).

Digital Object Identifier 10.1109/TPEL.2013.2251006

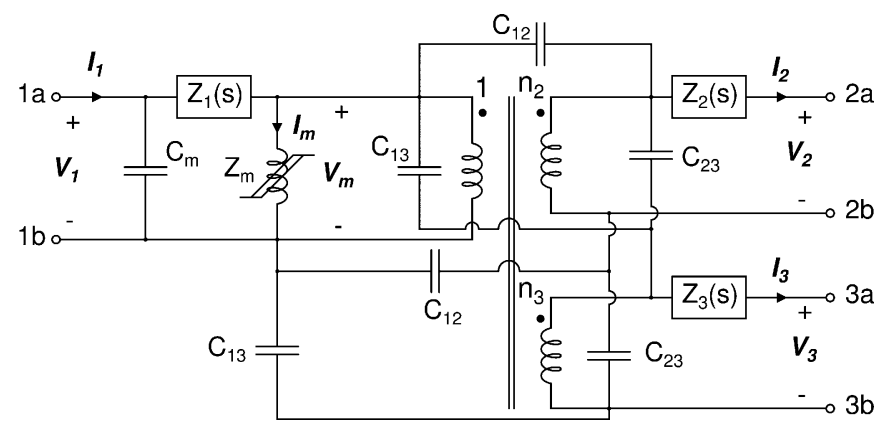

Fig. 1. Nonlinear dynamic model of a 3-winding transformer.

transformer at the same time, their models are obtained from an analytical [13], [14] or numerical [15] approach, i.e., based on physical equations or finite-element analysis, rather than a measurement-based one. There is, to the best of our knowledge, no investigation that tries to model both nonlinear and winding effects together using experimental data and system identification.

This paper is aimed at identifying and simulating a nonlinear dynamic model (including winding configuration properties and core characteristics) of a 3-winding transformer which is typically employed in a flyback converter. The equivalent model, as shown in Fig. 1, is generalized from the T-model for a 3-winding transformer [2]. The core behavior, consisting of a hysteresis effect and a nonlinear magnetic inductance, is characterized by $Z_{m}$. The impedance $\left\{Z_{i}(s)\right\}_{i=1}^{3}$, which represents the effect of the parasitic components in the $i$ th winding, is generally defined by

$$
Z_{i}(s)=R_{i}(s)+s L_{i}(s), i=1,2,3
$$

where $\left\{R_{i}(s)\right\}_{i=1}^{3}$ and $\left\{L_{i}(s)\right\}_{i=1}^{3}$ represent the power losses and the leakage inductance in the $i$ th winding, respectively. As both $\left\{R_{i}(s)\right\}_{i=1}^{3}$ and $\left\{L_{i}(s)\right\}_{i=1}^{3}$ are the functions of frequency, the winding impedance $\left\{Z_{i}(s)\right\}_{i=1}^{3}$ does not have a fixed form and depends on the configuration of each transformer. The capacitor $C_{m}$ describes the electric energy storage in all windings referred to the primary winding, while the electric energy storage between windings is symbolized by $C_{12}, C_{13}$, and $C_{23}$. The transformer voltage gains $n_{2}$ and $n_{3}$ account for the coupling between transformer windings. The capacitances $C_{m}, C_{12}, C_{13}$, and $C_{23}$ and the voltage gains $n_{2}$ and $n_{3}$ are presumed constant with frequency, in this study. The model in Fig. 1 is identified from two steps. First, the winding parameters are obtained using a small excitation signal, while the core model is estimated separately using a high-voltage source. 


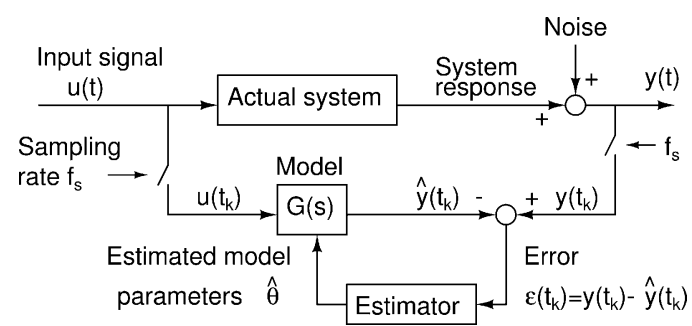

Fig. 2. Generic procedure to estimate a model of an actual system from sampled data.

For low-frequency applications, transformer winding model estimation has been widely studied using both time- and frequency-domain data [3], [4]. However, the studies for highfrequency transformers have been carried out in the frequency domain only [5]-[7]. The reason behind this selection is due to the advantages of data collection in frequency domain (by an impedance analyzer) over time domain (by an oscilloscope). In particular, for systems having wide variations in their frequency response, the collected time response data typically suffer from a round-off error due to the finite bit resolution of the acquisition device. Despite these limitations, the time-domain approach is still preferred as it requires only a simple measurement facility and offers an easy way to deal with a complex transformer model [3]. In the next section, a methodology is suggested to handle the numerical difficulties occurring during the measurement, and to obtain a frequency-dependent winding model of a transformer using system identification techniques.

A variety of modeling techniques have been suggested to emulate the hysteresis properties in the magnetic material [8]-[12]. Among them, the Jile-Atherton (J-A) model [8], [9], which offers the best tradeoff between simplicity and accuracy, can be considered as the most suitable approach for our application. Due to the effect of the air gap, the gap length is also considered as a variable during the estimation of the $\mathrm{J}-\mathrm{A}$ model.

The remainder of the paper is organized as follows. Section II introduces two nominal approaches to continuous-time system identification, while the data collection and application of these identification algorithms to transformer winding model are examined in Section III. The core model is described in Section IV. The flyback transformer is identified and verified in Section V. Conclusions are drawn in Section VI

\section{System IDENTIFICATION METHODS}

System identification has been widely applied in various fields to seek mathematical models of actual systems from their input-output behavior. As illustrated in Fig. 2, a typical system identification procedure requires a set of input-output data $\left\{u\left(t_{k}\right), y\left(t_{k}\right)\right\}_{k=1}^{N}$, a model structure (i.e., black box, gray box, etc.), and an estimation technique (i.e., least squares, maximum likelihood, etc.). For each model structure, model parameters are obtained by minimizing the error between the output signal $\left\{\hat{y}\left(t_{k}\right)\right\}_{k=1}^{N}$ predicted by the model and the actual output signal $\left\{y\left(t_{k}\right)\right\}_{k=1}^{N}$. A dynamic system model can be in discrete-time or continuous-time form. A high-fidelity continuous-time model is of primary interest in this paper.
Identification methods for a continuous-time system are typically classified as: 1) a direct approach which handles a continuous-time model of the system directly and 2) an indirect approach, in which a discrete-time model of the system is first identified and is then transformed into continuous-time form [16].

\section{A. Direct Continuous-Time Model Identification}

Due to the continuous-time nature of the transformer model in Fig. 1, a direct identification procedure is perfectly suitable to estimate transformer parameters. Various methods can be applied to the transformer model estimation problem [16]. However, only the most reliable method, denoted the simplified refined instrumental variable method for continuous-time system identification (SRIVC) [17], is chosen here. Let the continuous-time model of the actual system be

$$
G(s)=\frac{B_{c}(s)}{A_{c}(s)}=\frac{b_{n_{b}} s^{n_{b}}+b_{n_{b}-1} s^{n_{b}-1}+\cdots+b_{0}}{s^{n_{a}}+a_{n_{a}-1} s^{n_{a}-1}+\cdots+a_{0}} .
$$

Since only causal systems are considered, the transfer function $G(s)$ must be proper (i.e., $n_{b} \leq n_{a}$ ). The prediction error between the real and predicted output signals can be written as

$$
\begin{aligned}
\varepsilon(t) & =y(t)-\hat{y}(t)=y(t)-\frac{B_{c}(s)}{A_{c}(s)} u(t) \\
& =\frac{A_{c}(s)}{A_{c}(s)} y(t)-\frac{B_{c}(s)}{A_{c}(s)} u(t) .
\end{aligned}
$$

Let us assume that $A_{c}(s)$ in (3) is known; hence, $L(s)=$ $\frac{1}{A_{c}(s)}$ can be considered as a low-pass filter. Substituting filtered signals $y_{f}(t)=L(s) y(t)$ and $u_{f}(t)=L(s) u(t)$ into (3), we have

$$
\begin{aligned}
\varepsilon(t)= & A_{c}(s) y_{f}(t)-B_{c}(s) u_{f}(t) \\
= & y_{f}^{\left(n_{a}\right)}(t)+a_{n_{a}-1} y_{f}^{\left(n_{a}-1\right)}(t)+\cdots+a_{0} y_{f}(t) \\
& \quad-b_{n_{b}} u_{f}^{\left(n_{b}\right)}(t)-b_{n_{b}-1} u_{f}^{\left(n_{b}-1\right)} \cdots-b_{0} u_{f}(t)
\end{aligned}
$$

where

$$
\left\{\begin{array}{l}
y_{f}^{(i)}(t)=\mathcal{L}^{-1}\left\{\frac{s^{i}}{A_{c}(s)}\right\} * y(t), \quad i=0, \ldots, n_{a} \\
u_{f}^{(j)}(t)=\mathcal{L}^{-1}\left\{\frac{s^{j}}{A_{c}(s)}\right\} * u(t), \quad j=0, \ldots, n_{b} .
\end{array}\right.
$$

Here, the symbol $\mathcal{L}^{-1}$ and $*$ denote the inverse Laplace transform and convolution, respectively. By evaluating (4) at sampling instants $\left\{t_{k}\right\}_{k=1}^{N}$, we have $N$ equations in $n_{a}+n_{b}+1$ variables, which are the model parameters $\theta=$ $\left(a_{0}, a_{1}, \ldots, a_{n_{a}-1}, b_{0}, b_{1}, \ldots, b_{n_{b}}\right)$. If $N>n_{a}+n_{b}+1$, an unbiased solution of the model parameters can be found by a recursive instrumental variable algorithm. A detailed investigation of the SRIVC technique is documented in [17]. The implementation of the SRIVC method is available in the CONTSID toolbox for MATLAB [18]. 


\section{B. Indirect Continuous-Time Model Identification}

Though the indirect identification of a continuous-time model contains some potential issues induced by the discretization of a continuous-time system [16], this approach has been successfully applied in various applications, including the transformer model determination [3], [4]. For the comparison purpose, a discrete-time system identification algorithm based on a linear least-squares estimator (LSE) is presented. It is presumed that the discrete-time model of the actual system is given by

$$
G(z)=\frac{B_{d}(z)}{A_{d}(z)}=\frac{\beta_{0}+\beta_{1} z^{-1}+\cdots+\beta_{n_{\beta}} z^{-n_{\beta}}}{1+\alpha_{1} z^{-1}+\cdots+\alpha_{n_{\alpha}} z^{-n_{\alpha}}} .
$$

Due to the negative power representation of the variable $z$ in (5), the system $G(z)$ is always causal irrespective of the $n_{\alpha}$ and $n_{\beta}$ values. The signal $\hat{y}\left(t_{k}\right)$ can be estimated from the past observation of the input signal $\left\{u\left(t_{h}\right)\right\}_{t_{h} \leq t_{k}}$ and output signal $\left\{y\left(t_{l}\right)\right\}_{t_{l} \leq t_{k}-1}$ as

$$
\hat{y}\left(t_{k}\right)=\left(1-A_{d}(z)\right) y\left(t_{k}\right)+B_{d}(z) u\left(t_{k}\right)
$$

or in matrix form

$$
\hat{y}\left(t_{k}\right)=\varphi\left(t_{k}\right) \theta^{T}
$$

where the vector $\theta=\left[\alpha_{1} \alpha_{2} \ldots \alpha_{n_{\alpha}} \beta_{0} \beta_{1} \ldots \beta_{n_{\beta}}\right]$ contains the model parameters and the vector

$$
\begin{aligned}
\varphi\left(t_{k}\right)=\left[-y\left(t_{k}-1\right)-y\left(t_{k}-2\right) \ldots-y\left(t_{k}-n_{\alpha}\right)\right. \\
\left.u\left(t_{k}\right) u\left(t_{k}-1\right) \ldots u\left(t_{k}-n_{\beta}\right)\right] .
\end{aligned}
$$

The prediction error at time instance $t_{k}\left(\max \left(n_{\alpha}, n_{\beta}\right)<t_{k} \leq\right.$ $N)$ is

$$
\varepsilon\left(t_{k}\right)=y\left(t_{k}\right)-\hat{y}\left(t_{k}\right)=y\left(t_{k}\right)-\varphi\left(t_{k}\right) \theta^{T} .
$$

The lower bound of $t_{k}$ is defined to make sure that all past input and output samples in $\varphi\left(t_{k}\right)$ of (8) are available. If the prediction error $\left\{\varepsilon\left(t_{k}\right)\right\}_{t_{k}=\max \left(n_{\alpha}, n_{\beta}\right)+1}^{N}$ is white noise, an estimate of the model parameters $\theta$ can be solved using the least squares as

$$
\hat{\theta}=\left[\Phi_{N_{\alpha}}^{T} \Phi_{N_{\alpha}}\right]^{-1} \Phi_{N_{\alpha}}^{T} Y_{N_{\alpha}}
$$

where $Y_{N_{\alpha}}=\left[y\left(n_{\alpha}+1\right) y\left(n_{\alpha}+2\right) \ldots y(N)\right]^{T}$ is the output vector and $\Phi_{N_{\alpha}}=\left[\varphi^{T}\left(n_{\alpha}+1\right) \varphi^{T}\left(n_{\alpha}+2\right) \ldots \varphi^{T}(N)\right]^{T}$ denotes the regressor matrix. In the case of a colored prediction error, the instrumental variable method and/or a noise model is typically employed to avoid a biased estimation of $\theta$ [19].

By applying a discrete-to-continuous (D2C) time transformation to $G(z)$, one can determine a continuous-time model $G(s)$ of the actual system. Though various mappings between the discrete and continuous time domain are available, only the Tustin's transformation is considered to preserve the frequency response of the system with resemble fidelity. One limitation of indirect system identification is the nonpreservation of model order during a D2C transformation. This is partly due to $n_{a}, n_{b}$ in (2) and $n_{\alpha}, n_{\beta}$ in (5) have different roles in describing system properties. The nonpreserved model order implies that we cannot control the order of $G(s)$ by only specifying the order of $G(z)$. However, the order of $G(s)$ can be retained by putting a constraint on the model parameters of $G(z)$. Starting with $G(s)$ described by (2), discretizing $G(s)$ using the Tustin's transformation $s=\frac{2}{T} \frac{1-z^{-1}}{1+z^{-1}}$, we have

$$
G(z)=\frac{b_{n_{b}}\left(\frac{2}{T} \frac{1-z^{-1}}{1+z^{-1}}\right)^{n_{b}}+b_{n_{b}-1}\left(\frac{2}{T} \frac{1-z^{-1}}{1+z^{-1}}\right)^{n_{b}-1}+\cdots+b_{0}}{\left(\frac{2}{T} \frac{1-z^{-1}}{1+z^{-1}}\right)^{n_{a}}+a_{n_{a}-1}\left(\frac{2}{T} \frac{1-z^{-1}}{1+z^{-1}}\right)^{n_{a}-1}+\cdots+a_{0}} .
$$

We multiply both numerator and denominator of $G(z)$ by $\left(1+z^{-1}\right)^{n_{a}}$ and rearrange the result to the form

$$
\begin{aligned}
G(z) & =\left(1+z^{-1}\right)^{n_{a}-n_{b}}\left[\frac{\beta_{0}+\beta_{1} z^{-1}+\cdots+\beta_{n_{b}} z^{-n_{b}}}{1+\alpha_{1} z^{-1}+\cdots+\alpha_{n_{a}} z^{-n_{a}}}\right] \\
& =\left(1+z^{-1}\right)^{n_{a}-n_{b}} G^{*}(z)
\end{aligned}
$$

where

$$
G^{*}(z)=\frac{\beta_{0}+\beta_{1} z^{-1}+\cdots+\beta_{n_{b}} z^{-n_{b}}}{1+\alpha_{1} z^{-1}+\cdots+\alpha_{n_{a}} z^{-n_{a}}}
$$

and $\beta_{0}, \beta_{1}, \ldots, \beta_{n_{b}}$ are a linear combination of $b_{0}, b_{1}, \ldots, b_{n_{b}}$ and $\alpha_{1}, \alpha_{2}, \ldots, \alpha_{n_{a}}$ are a linear combination of $a_{0}, a_{1}, \ldots, a_{n_{a}-1}$. It can be demonstrated that when the parameters $a_{0}, a_{1}, \ldots, a_{n_{a}-1}$ and $b_{0}, b_{1}, \ldots, b_{n_{b}}$ are independent, $\beta_{0}, \beta_{1}, \ldots, \beta_{n_{b}}$ and $\alpha_{1}, \alpha_{2}, \ldots, \alpha_{n_{a}}$ are also independent. This means that there is no constraint on the parameters of $G^{*}(z)$, and that the numerator order $n_{b}$ and denominator order $n_{a}$ of $G(s)$ can always be preserved if $G(z)$ satisfies (11) and Tustin's transformation is used. Identifying $G(z)$ from the input-output data $\left\{u\left(t_{k}\right), y\left(t_{k}\right)\right\}$ now reduces to the estimation $G^{*}(z)$ from the filtered data $\left\{u\left(t_{k}\right)\left(1+z^{-1}\right)^{n_{a}-n_{b}}, y\left(t_{k}\right)\right\}$ and then applying (11). It should be noted that the idea of using a prefilter to establish a unique mapping between parameters of continuoustime model and its equivalent discrete time has been exploited somewhere in [20]. However, the study in [20] can only be applied when the model structure is known and simple.

\section{TRANSFORMER WINDING MODEL IDENTIFICATION}

\section{A. Data Collection}

Time-domain data can be collected in many ways depending on the algorithm used to estimate the transformer model and the specifications of the measurement equipment [3], [4]. Since the transformer parameters in Fig. 1 generally exhibit different orders of magnitude, it is preferable to determine them separately using different sets of input/output data [4]. This can be achieved by running different experiments, each of which is configured such that the response of some model parameters is dominant in the collected data while the effect of other parameters can be neglected. Though various experimental configurations, for example short circuiting the transformer winding, removing the ferrite core, using band-limited excitation signals, etc., have been studied in [4], [6], and [7], their performance is dependent on the transformer under test. For simplicity, each experiment in this paper is carried out by a random excitation signal in combination with a particular short circuiting arrangement.

A circuit arrangement used to obtain time-series data for an experiment is exemplified in Fig. 3. The transformer is simply depicted by the 3-port network $\mathbf{T}$. A random binary voltage 


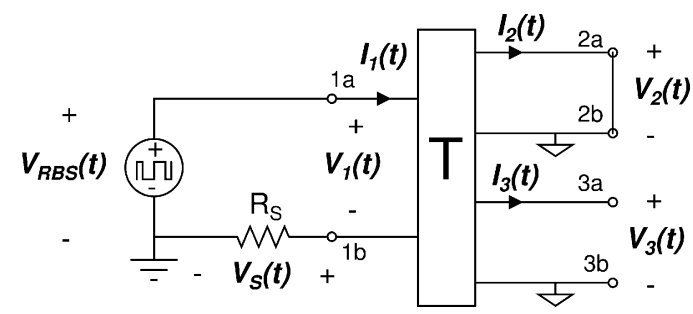

Fig. 3. Experimental configuration to collect input/output data for 3-winding transformer model estimation.

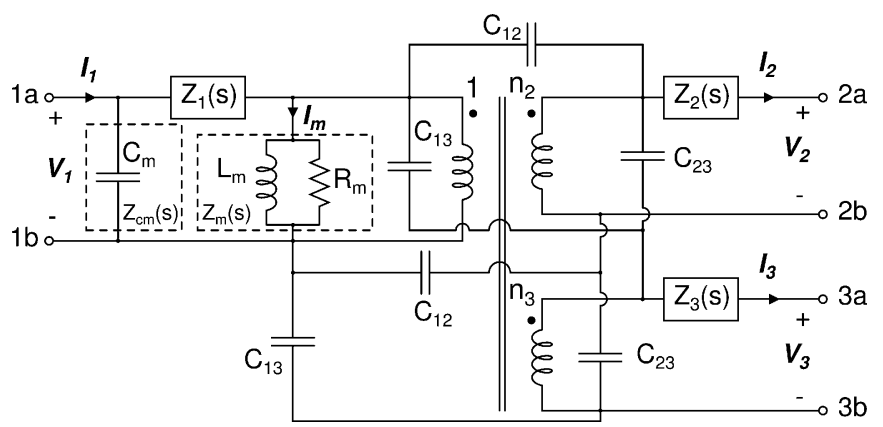

Fig. 4. Transformer winding model with a linear core.

$V_{\mathrm{RBS}}(t)$, which has a virtually flat spectrum over the frequency range of interest, is injected into the primary winding. The sensing resistor $R_{S}$ is deliberately inserted to provide a current measurement. Only a short circuited connection of the secondary winding is involved in this experiment. The data for system identification (e.g. $V_{\mathrm{RBS}}(t), V_{S}(t)$ and $V_{3}(t)$ ) are collected by a digital oscilloscope. These voltages can serve as either input or output data for system identification depending on the objective of the particular test. For other experiments, the excitation signal $V_{\mathrm{RBS}}(t)$ might be applied to other transformer terminals with different winding short circuit setup. However, the resistor $R_{S}$ is always kept in series with $V_{\mathrm{RBS}}(t)$.

The difficulties of time-domain identification methods arise when the response of the system, e.g., $V_{S}(t)$ and $V_{3}(t)$ in Fig. 3, varies significantly over a wide frequency range and cannot be sufficiently resolved by the digital oscilloscope. The roundoff error occurring will distort the estimated result regardless of the identification techniques used. Two options, consisting of increasing the resolution of each sample (more expensive measurement equipment), and considering only the dynamic response of interest, can be used to reduce the round-off error. The latter approach will be used in this paper to enrich some dynamic contents in $V_{S}(t)$ by adjusting $R_{S}$, which plays the same role as a ranging resistor in impedance measurement circuits [21].

\section{B. Parameter Estimation Procedure}

Under small signal excitation, the nonlinear core model in Fig. 1 is presumed linear and can be modeled by a parallel $R_{m} L_{m}$ circuit, as shown in Fig. 4. A set of six different experiments is performed to derive all the parameters for the winding model in Fig. 4. To clarify the measurement and identification procedure, a fixed template for each experiment, described later, is introduced.

1) Experimental configuration specification: It describes how to set up the measurement circuit for each experiment. The information in this item includes the transformer terminals to which the voltage $V_{\mathrm{RBS}}(t)$ is applied, the sensing resistor value $R_{S}$ and the short circuit connection between transformer terminals. For example, $A T_{1 a 1 b}, R_{S}=2 \Omega$, and $S_{2 a 2 b}$ means that $V_{\mathrm{RBS}}$ is injected to the terminals $1 a$ and $1 b$ of the transformer, the value of the sensing resistor is $2 \Omega$, and a short circuit is made between terminals $2 a$ and $2 b$.

2) Data collection: It specifies which signal in the experimental circuit will be captured by the oscilloscope.

3) Identification objective: It denotes the transfer function and model parameters obtained from measured data.

Experiment 1:

1) $A T_{1 a 1 b}, R_{S}=465.5 \Omega$, no short connection.

2) $V_{\mathrm{RBS}}, V_{S}$.

3) $\frac{Z_{c m}(s) Z_{m}(s)}{Z_{c m}(s)+Z_{m}(s)}, R_{m}, L_{m}, C_{m}$.

Experiment 2:

1) $A T_{1 a 1 b}, R_{S}=6.84 \Omega, S_{2 a 2 b}$.

2) $V_{\mathrm{RBS}}, V_{S}, V_{3}$.

3) $Z_{1}(s), Z_{2}(s)$.

Experiment 3:

1) $A T_{1 a 1 b}, R_{S}=6.84 \Omega, S_{3 a 3 b}$.

2) $V_{\mathrm{RBS}}, V_{S}, V_{2}$.

3) $Z_{1}(s), Z_{3}(s)$.

Experiment 4:

1) $A T_{1 a 2 a}, R_{S}=465.5 \Omega, S_{1 a 1 b}, S_{2 a 2 b 3 a 3 b}$.

2) $V_{\mathrm{RBS}}, V_{S}$.

3) $\frac{2 R_{S}\left(C_{12}+C_{13}\right) s}{2 R_{S}\left(C_{12}+C_{13}\right) s+1}, C_{12}+C_{13}$.

Experiment 5:

1) $A T_{1 a 2 a}, R_{S}=465.5 \Omega, S_{1 a 1 b 3 a 3 b}, S_{2 a 2 b}$.

2) $V_{\mathrm{RBS}}, V_{S}$.

3) $\frac{2 R_{S}\left(C_{12}+C_{23}\right) s}{2 R_{S}\left(C_{12}+C_{23}\right) s+1}, C_{12}+C_{23}$.

Experiment 6:

1) $A T_{2 a 3 a}, R_{S}=465.5 \Omega, S_{1 a 1 b 2 a 2 b}, S_{3 a 3 b}$.

2) $V_{\mathrm{RBS}}, V_{S}$.

3) $\frac{2 R_{S}\left(C_{23}+C_{13}\right) s}{2 R_{S}\left(C_{23}+C_{13}\right) s+1}, C_{23}+C_{13}$.

The voltage transform ratios are computed as $n_{2}=\frac{N_{2}}{N_{1}}$ and $n_{3}=\frac{N_{3}}{N_{1}}$, where $N_{1}, N_{2}$, and $N_{3}$ are the number of turns in first, second, and third windings, respectively. In order to explain how to obtain the model parameters in Fig. 4 from each experiment, the following assumptions are made.

1) Interwinding capacitances $C_{12}, C_{23}$, and $C_{13}$ can be identified separately from the rest of the winding model.

2) Impedance $Z_{m}(s)$ is typically much larger than $Z_{1}(s)$; hence, we can neglect $Z_{1}(s)$ in Experiment 1 .

3) Impedance of $C_{m}$, say $Z_{c m}(s)$, is much higher than $Z_{1}(s), Z_{2}(s)$, and $Z_{3}(s)$ in the frequency range under investigation; therefore, $Z_{c m}(s)$ is ignored in Experiments 2 and 3.

Given the measurement setup and assumption for Experiment 1 , one can establish the relation between the measured voltages 
$V_{\mathrm{RBS}}(t)$ and $V_{S}(t)$ as

$$
V_{\mathrm{RBS}}(t)-V_{S}(t)=\frac{Z_{c m}(s) Z_{m}(s)}{Z_{c m}(s)+Z_{m}(s)} \frac{V_{S}(t)}{R_{S}} .
$$

Equation (12) shows that by applying the techniques in Section II with input data $V_{S}(t) / R_{S}$ and output data $V_{\mathrm{RBS}}(t)-$ $V_{S}(t)$, one can estimate the transfer function $\frac{Z_{c m}(s) Z_{m}(s)}{Z_{c m}(s)+Z_{m}(s)}=$ $\frac{R_{m} L_{m} s}{R_{m} L_{m} C_{m} s^{2}+L_{m} s+R_{m}}$ which is a basis for the determination of $R_{m}, L_{m}$, and $C_{m}$.

For Experiment 2, the identification of the impedances $Z_{1}(s)$ and $Z_{2}(s)$ requires an extra processing step. Let us start from (13) which relates the collected signals $V_{3}(t)$ and $V_{S}(t)$ with transformer parameters by

$$
V_{3}(t)=n_{3} \frac{Z_{2}(s) Z_{m}(s)}{Z_{2}(s)+n_{2}^{2} Z_{m}(s)} \frac{V_{S}(t)}{R_{S}} .
$$

Divide both sides of (13) by $\frac{Z_{2}(s) Z_{m}(s)}{Z_{2}(s)+n_{2}^{2} Z_{m}(s)}$ and rearrange the result as

$$
n_{3}\left(\frac{V_{S}(t)}{R_{S}}-\hat{I}_{m}(t)\right)=\frac{1}{Z_{2}(s)} n_{2}^{2} V_{3}(t)
$$

where

$$
\hat{I}_{m}(t)=\frac{1}{n_{3} Z_{m}(s)} V_{3}(t) .
$$

Since $Z_{m}(s)$ is available from Experiment 1, we can easily calculate $\hat{I}_{m}(t)$ from $V_{3}(t)$ via (15). Let us assume that $Z_{2}(s)$ is not proper. Using $n_{2}^{2} V_{3}(t)$ and $n_{3}\left(V_{S}(t) / R_{S}-\hat{I}_{m}(t)\right)$ as input and output data for system identification, respectively, one can obtain $1 / Z_{2}(s)$ according to (14) and $Z_{2}(s)$ through the inversion of $1 / Z_{2}(s)$. In the case of a proper impedance $Z_{2}(s)$, by interchanging the input-output role of $n_{2}^{2} V_{3}(t)$ and $n_{3}\left(V_{S}(t) / R_{S}-\hat{I}_{m}(t)\right)$ during an identification step, an estimation of $Z_{2}(s)$ can be achieved. For estimation of $Z_{1}(s)$, one can rely upon (16) and the data $V_{\mathrm{RBS}}(t), V_{S}(t)$, and $V_{3}(t)$ acquired in Experiment 2, via

$$
\frac{V_{S}(t)}{R_{S}}=\frac{1}{Z_{1}(s)}\left(V_{\mathrm{RBS}}(t)-V_{S}(t)-\frac{V_{3}(t)}{n_{3}}\right) .
$$

The determination of the impedances $Z_{1}(s)$ and $Z_{3}(s)$ in Experiment 3 can be carried out in a similar way as implemented in Experiment 2. Since the impedance $Z_{1}(s)$ can be obtained from either Experiment 2 or 3, the similarity of the two results will act as a validation for the estimation technique.

As the same procedure can be used to determine model parameters from captured data in Experiments 4-6, hence only consideration for Experiment 4 is taken into account. Thanks to the effect of the circuit layout in Experiment 4, only capacitors $C_{12}$ and $C_{13}$ are involved in shaping the voltage $V_{S}(t)$ by

$$
V_{S}(t)=\frac{2 R_{S}\left(C_{12}+C_{13}\right) s}{2 R_{S}\left(C_{12}+C_{13}\right) s+1} V_{\mathrm{RBS}}(t) .
$$

It is obvious from (17) that when the signals $V_{\mathrm{RBS}}(t)$ and $V_{S}(t)$ are known, one can easily work out the value of $C_{12}+C_{13}$. Combining the results from Experiments 4-6, there exists a unique solution for $C_{12}, C_{13}$, and $C_{23}$.
In addition to the input-output data, a properly chosen order for each transfer function is a prerequisite for the system identification procedure. Fortunately, the transfer function order can be computed based on the impedance of the model parameters that we want to determine in each experiment. For the constant parameters, say $L_{m}, R_{m}, C_{m}, C_{12}, C_{23}$, and $C_{13}$, their corresponding impedances have a fixed order. By contrast, the impedances $Z_{1}(s), Z_{2}(s)$, and $Z_{3}(s)$ of the frequencydependent parameters have an undefined order, as shown by

$$
\begin{aligned}
Z_{i}(s) & =R_{i}(s)+s L_{i}(s) \\
& =\frac{b_{i 1} s^{n_{i b}-1}+b_{i 2} s^{n_{i b}-2}+\cdots+b_{i n_{i b}}}{s^{n_{i a}}+a_{i 1} s^{n_{i a}-1}+\cdots+a_{i n_{i a}}}, i=1,2,3 .
\end{aligned}
$$

The general description of $\left\{Z_{i}(s)\right\}_{i=1}^{3}$ in (18) is suitable for the identification step but difficult to implement in electrical simulators. This problem can be solved by constraining the parameters of (18) so that $\left\{Z_{i}(s)\right\}_{i=1}^{3}$ can be represented by Foster's network [6]. In most cases, we found that a simple condition between $\left\{n_{i b}\right\}_{i=1}^{3}$ and $\left\{n_{i a}\right\}_{i=1}^{3}$, as shown in (19), is enough to obtain $\left\{Z_{i}(s)\right\}_{i=1}^{3}$ with Foster's network form

$$
n_{i b}=n_{i a}+2, i=1,2,3 \text {. }
$$

The values of $\left\{n_{i b}\right\}_{i=1}^{3}$ in both Experiments 2 and 3 are obtained using a singular value decomposition (SVD) [19]. The result from the SVD is consistent with that from both the Akaike information criterion and minimum description length [19], though these complexity weightings give a less definitive selection.

\section{NONLINEAR CORE MOdEL IDENTIFICATION}

The nonlinear effect of the ferrite core is represented by modeling the magnetizing inductance $Z_{m}$ in Fig. 1 as a nonlinear inductor. Applying the $\mathrm{J}-\mathrm{A}$ model [8], the current $I_{m}$ passing through $Z_{m}$ can be computed from the voltage $V_{m}$ across it, via

$$
\begin{aligned}
& \frac{d B}{d t}=\frac{V_{m}}{N_{1} A_{c}} \\
& H=\frac{B}{\mu_{0}}-M \\
& H_{e}=H+\alpha M \\
& M_{\mathrm{an}}=M_{s}\left[1-\operatorname{coth}\left(\frac{H_{e}}{a}\right)+\left(\frac{a}{H_{e}}\right)\right] \\
& \frac{d M_{\mathrm{an}}}{d H_{e}}=\frac{M_{s}}{a}\left[1-\operatorname{coth} h^{2}\left(\frac{H_{e}}{a}\right)+\left(\frac{a}{H_{e}}\right)^{2}\right] \\
& \frac{d M_{\mathrm{irr}}}{d H_{e}}=\frac{\gamma\left(M_{\mathrm{an}}-M_{\mathrm{irr}}\right)}{k \delta} \\
& \delta=\left\{\begin{array}{ll}
1 & \text { if } \frac{d H}{d t} \geq 0 \\
-1 & \text { if } \frac{d H}{d t}<0
\end{array} \quad \gamma= \begin{cases}1 & \text { if } M_{\text {an }}-M_{\text {irr }} \geq 0 \\
0 & \text { if } M_{\text {an }}-M_{\text {irr }}<0\end{cases} \right. \\
& \frac{d M}{d B}=\mu_{0} \frac{(1-c) \frac{d M_{\mathrm{irr}}}{d H_{e}}+c \frac{d M_{\mathrm{an}}}{d H_{e}}}{1+(1-\alpha) c \frac{d M_{\mathrm{an}}}{d H_{e}}+(1-\alpha)(1-c) \frac{d M_{\mathrm{irr}}}{d H_{e}}}
\end{aligned}
$$


TABLE I

WINDING MODEL PARAMETERS

\begin{tabular}{|c|c|c|}
\hline Component & SRIVC Algorithm & LSE Algorithm \\
\hline$L_{m}$ & $791.67 \mu H$ & $791.62 \mu H$ \\
\hline$R_{m}$ & $85.522 k \Omega$ & $85.078 k \Omega$ \\
\hline$C_{m}$ & $8.9903 p F$ & $8.9065 p F$ \\
\hline$C_{12}$ & $3.298 p F$ & $3.233 p F$ \\
\hline$C_{13}$ & $59.64 p F$ & $59.58 p F$ \\
\hline$C_{23}$ & $29.304 p F$ & $29.235 p F$ \\
\hline$n_{2}$ & 0.21739 & 0.21739 \\
\hline$n_{3}$ & 0.13043 & 0.13043 \\
\hline$Z_{1}(s)$ & $0.3522+9.745 \cdot 10^{-7} s+\frac{1.307 s}{s+5.692 \cdot 10^{6}}$ from Experiment 2 & $0.3546+9.7037 \cdot 10^{-7} s+\frac{1.372 s}{s+5.927 \cdot 10^{6}}$ from Experiment 2 \\
& $0.3485+9.636 \cdot 10^{-7} s+\frac{1.49 s}{s+6.127 \cdot 10^{6}}$ from Experiment 3 & $0.3501+9.596 \cdot 10^{-6} s+\frac{1.504 s}{s+6.334 \cdot 10^{6}}$ from Experiment 3 \\
\hline$Z_{2}(s)$ & $0.0422+1.246 \cdot 10^{-7} s+\frac{0.0877 s}{s+3.996 \cdot 10^{6}}$ & $0.0895 s$ \\
\hline$Z_{3}(s)$ & $0.0425+7.525 \cdot 10^{-8} s+\frac{0.0364 s}{s+2.448 \cdot 10^{6}}$ & $0.0422+1.245 \cdot 10^{-7} s+\frac{0.0635+10}{s+4.0635 \cdot 10^{6}}$ \\
\hline
\end{tabular}

$$
\begin{aligned}
\frac{d M}{d t} & =\frac{d M}{d B} \frac{d B}{d t} \\
I_{m} & =\frac{1}{N_{1}}\left[H\left(l_{e}-l_{a}\right)+\frac{B l_{a}}{\mu_{0}}\right]
\end{aligned}
$$

where $B$ and $H$ are the flux density and applied magnetic field inside the ferrite core, respectively. $M, M_{\mathrm{irr}}$, and $M_{\mathrm{an}}$ represent the total, irreversible, and anhysteresis magnetization quantities. The rate-dependent effect of the magnetic material has not considered herein, but could be incorporated to the given static model by adding an extra dynamic to the magnetization $M$ [9].

The nonlinear inductor model parameters include the primary winding turns $N_{1}$, effective magnetic path length $l_{e}$, air-gap length $l_{a}$, effective core area $A_{c}$, and J-A model parameters $\left(M_{s}, a, \alpha, c\right.$, and $\left.k\right)$. Apart from $N_{1}, l_{e}$, and $A_{c}$ which can be determined from a transformer core datasheet, the other parameter including the J-A model parameters and the gap length $l_{a}$ will be estimated using an optimization method. In particular, $M_{s}, a, \alpha, c, k$, and $l_{a}$ are selected to minimize the objective function in

$$
\varepsilon=\sum_{k=1}^{N} \frac{\left(I_{m \exp }(k)-I_{m \operatorname{sim}}(k)\right)^{2}}{N}
$$

where $N$ denotes the total number of data points, and $I_{m \exp }$ and $I_{m \operatorname{sim}}$ are the experimental and simulated inductor currents, respectively. Since both $\alpha$ and $l_{a}$ contribute to the slope of the hysteresis curve, the identification of the model parameters should be carried out in two stages. In the first step, the gap length $l_{a}$ is kept fixed, while the $\mathrm{J}-\mathrm{A}$ parameters are found using the differential evolution (DE) method [22]. All parameters obtained in the first step including $l_{a}$ are then used as an initial condition for the Nelder-Mead algorithm in the second step. We note that the value of $l_{a}$ in the first step can be read from the transformer design specification.

\section{EXPERIMENTAL RESULTS}

\section{A. Winding Model Determination and Validation}

The identification procedure, described in Section III, is applied to a 3-winding flyback transformer designed for a power supply application. The transformer operates with a nominal switching frequency of $100 \mathrm{kHz}$. The first, second, and

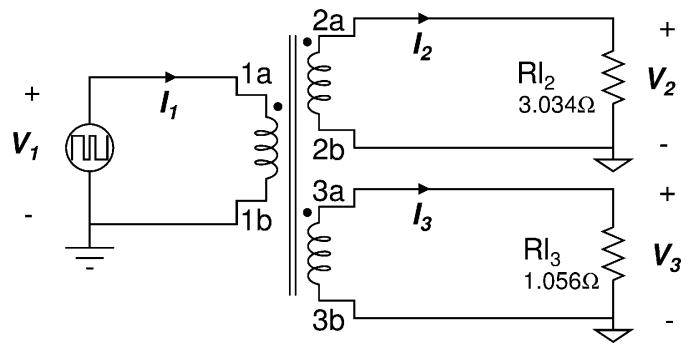

Fig. 5. Circuit structure for validating the transformer winding model in time domain.

third winding turns are 46,10, and 6, respectively. An E core (E25/13/7 type, EPCOS N87) with an air-gap length of approximately $0.15 \mathrm{~mm}$ is required to provide an expected inductance of $800 \mu \mathrm{H}$. A signal generator circuit is designed and implemented to provide a symmetrical random binary voltage $V_{\mathrm{RBS}}(t)$, having an adjustable amplitude of $0-2 \mathrm{~V}$, a clock of $5 \mathrm{MHz}$, and a flat spectrum from $100 \mathrm{~Hz}$ to $5 \mathrm{MHz}$. The input-output data are acquired by an Agilent digital oscilloscope (DSO6054A) with a preset sampling rate of $50 \mathrm{MHz}$ and 12 bits for each sample.

The estimated parameters of the winding model from both SRIVC and LSE methods in Section II are summarized in Table I, where the impedance $Z_{1}(s)$ obtained from Experiments 2 and 3 are both included for comparison. From Table I, the two independent estimates of $Z_{1}(s)$ are closely matched. This effectively validates our estimation approach. In addition, the results from SRIVC and LSE demonstrate that by imposing a proper constraint [see (11)] on the discrete time model, the LSE approach can perform, as well as the SRIVC method, in estimating a continuous time model.

Both time- and frequency-domain validations are essential to examine the accuracy of the obtained winding models. The circuit prototype, as shown in Fig. 5, is implemented to capture data for time-domain validation. A random binary voltage $V_{1}(t)$, a part of which is plotted in Fig. 6, is injected to the primary transformer. The corresponding secondary and third winding voltages, which are returned from the experiment and simulation, are compared in Fig. 6. The excellent fit between the measured and simulated signals strongly confirm the accuracy of the obtained model. The comparison with different load 

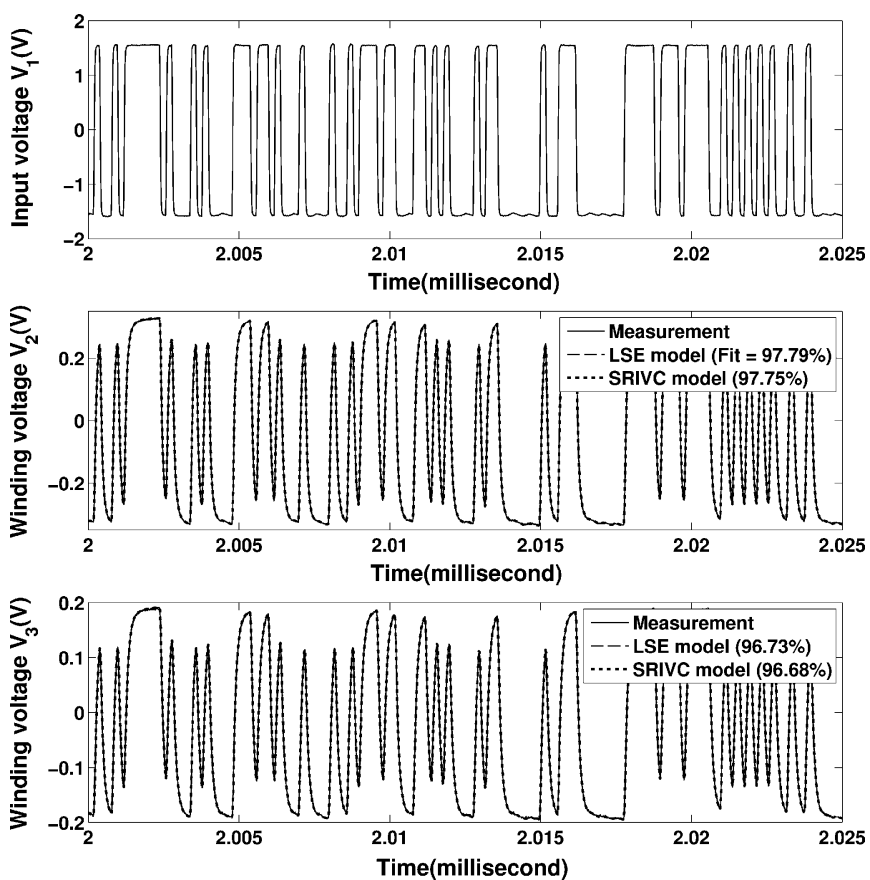

Fig. 6. Measured and simulated results obtained from the time-domain model validation scheme in Fig. 5.

TABLE II

FREQUENCY-DOMAIN TEST CONFIGURATIONS

\begin{tabular}{|c|c|}
\hline Test case & Winding configuration \\
\hline 1 & Open circuit both secondary and third winding \\
\hline 2 & $\begin{array}{r}\text { Open circuit secondary winding and } \\
\text { short circuit third winding }\end{array}$ \\
\hline 3 & $\begin{array}{r}\text { Short circuit secondary winding and } \\
\text { open circuit third winding }\end{array}$ \\
\hline
\end{tabular}

resistors, not presented here due to space limitation, also shows good agreement.

A frequency-domain test is performed using an HP4194A Impedance/Gain-Phase Analyzer. The primary impedance of the transformer is measured and calculated between $500 \mathrm{~Hz}$ and $15 \mathrm{MHz}$ in three different circuit configurations, as presented in Table II. The results for each configuration are, in turn, depicted in Figs. 7-9. In these figures, the estimated frequency response is obtained from the sampled voltage and current signals using spectral analysis [19], while the measured data are provided by the impedance analyzer. The estimated frequency response has a big transition at multiples clock frequency of $5 \mathrm{MHz}$. This is due to the excitation voltage (random binary signal), the spectrum of which typically has a notch at the clock frequency and its harmonics. When comparing the modeled, estimated, and measured frequency responses in the three test cases (see Table II), one can say that both models can accurately predict all dynamics of the real transformer over a wide frequency range. The only noticeable discrepancy between the measured and modeled results is shown in Fig. 9, when the frequency is smaller than $10 \mathrm{kHz}$. This offset is probably due to the error when measuring the primary impedance at low frequencies using the impedance analyzer.
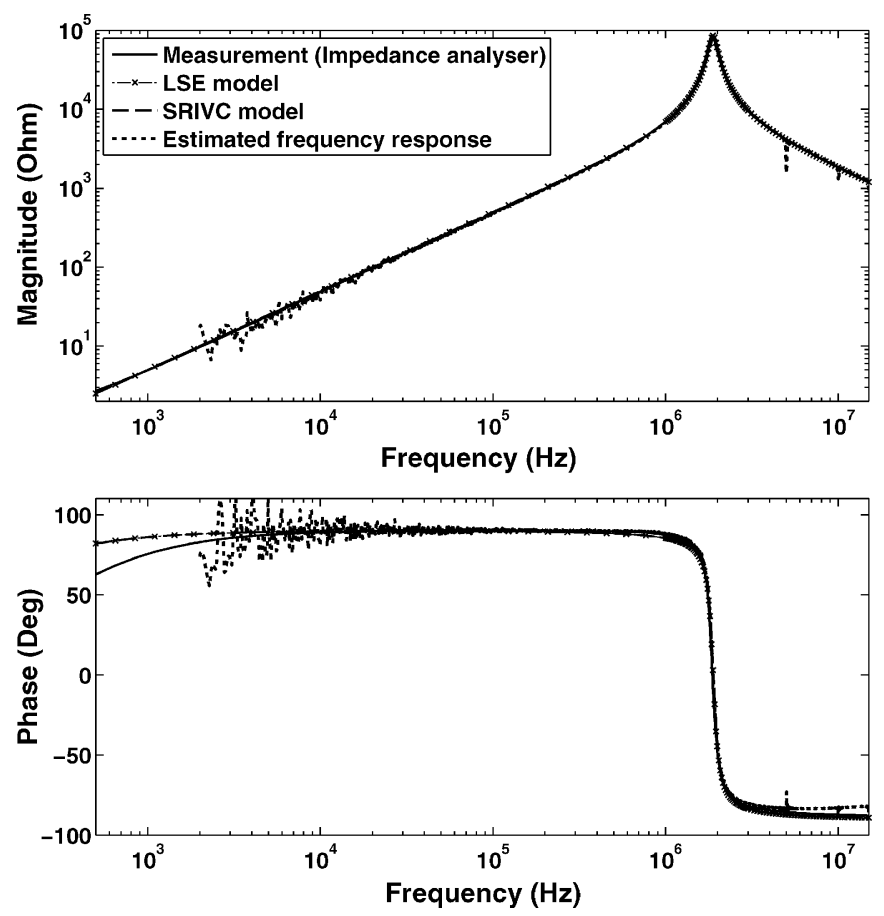

Fig. 7. Primary transformer impedance for test case 1 (open circuit secondary and third windings).
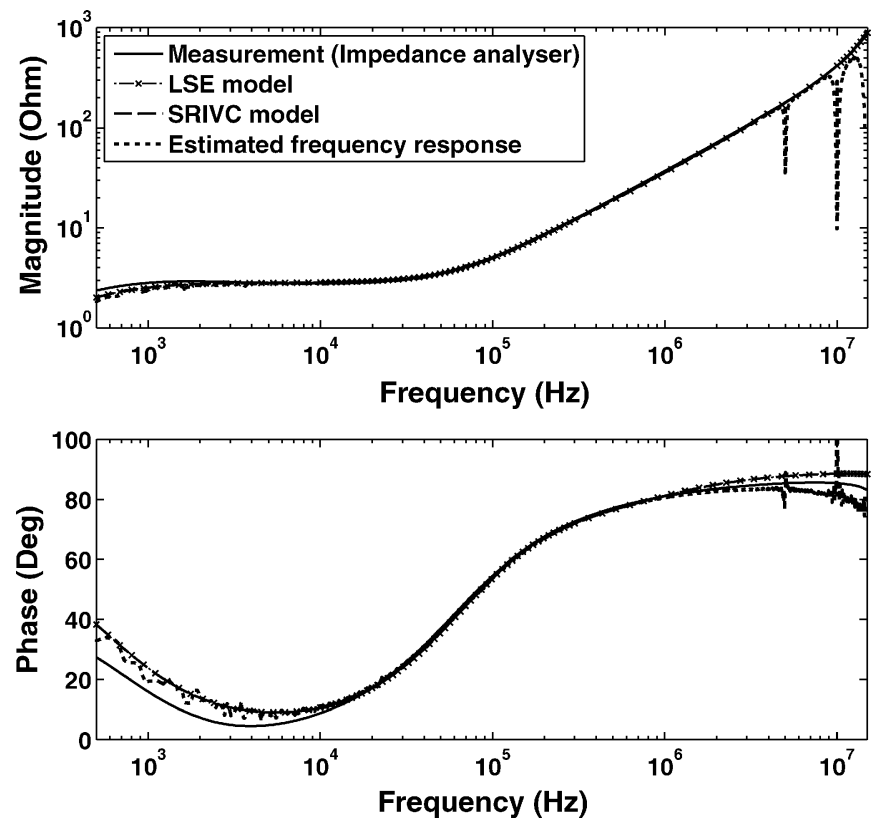

Fig. 8. Primary transformer impedance for test case 2 (open circuit secondary and short circuit third winding).

\section{B. Core Model}

The voltage $V_{m}$ and current $I_{m}$ data for the core model estimation in Section IV are obtained using the conventional test method for ring cores [10], [11] except that the sample under investigation is now the flyback transformer. Specifically, the excitation source to the primary winding is a sinusoidal voltage having a frequency of $1 \mathrm{kHz}$ and a variable amplitude of $2-$ $12.5 \mathrm{~V}$, while the data are measured using a digital oscilloscope 

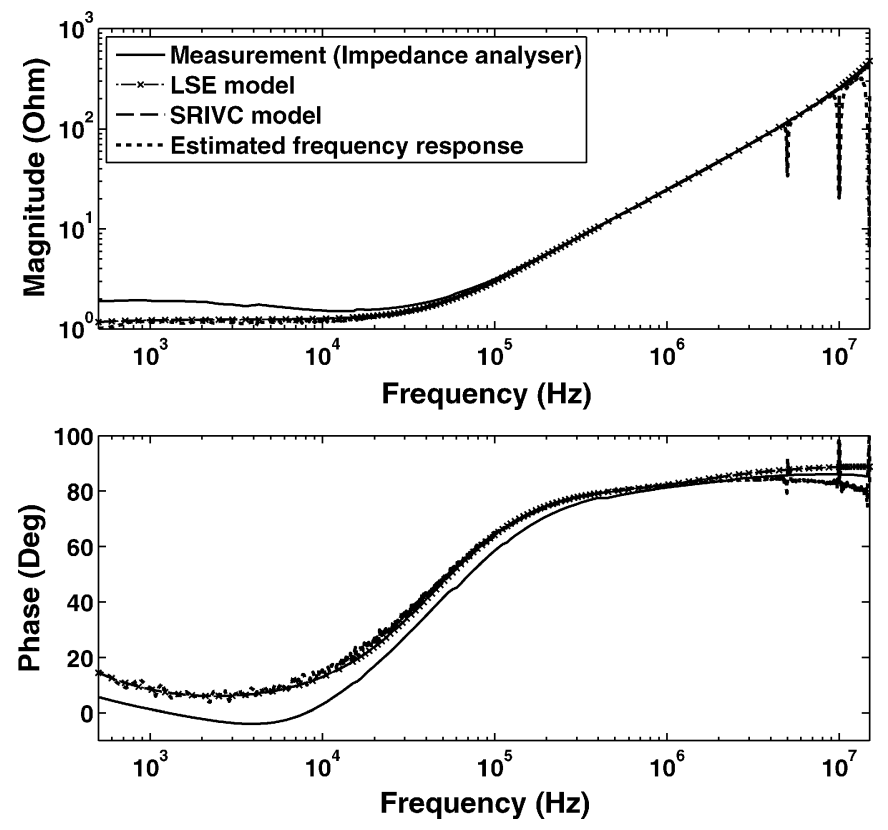

Fig. 9. Primary transformer impedance for test case 3 (short circuit secondary and open circuit third winding).

TABLE III

OPTIMIZED NONLINEAR INDUCTOR MODEL PARAMETERS

\begin{tabular}{|c|c|c|c|}
\hline Parameter & Initial value & DE & Nelder-Mead \\
\hline$M_{s}(A / m)$ & $10^{5}-10^{6}$ & $4.0204 \cdot 10^{5}$ & $4.025 \cdot 10^{5}$ \\
$a(A / m)$ & $5-500$ & 41.1123 & 41.7453 \\
$\alpha$ & $10^{-5}-10^{-3}$ & $2.1467 \cdot 10^{-4}$ & $2.1019 \cdot 10^{-5}$ \\
$c$ & $0.1-0.99$ & 0.5649 & 0.5751 \\
$k_{0}(A / m)$ & $5-500$ & 42.4702 & 43.4395 \\
$l_{a}(m m)$ & 0.15 & 0.15 & 0.1514 \\
\hline
\end{tabular}

at the secondary winding and the current sense resistor of $3.9 \Omega$. To ensure that the obtained model can work accurately over a wide variety of operating condition, different levels of $V_{m}$ and $I_{m}$ (corresponding to different hysteresis curves) are included in the optimization procedure. The optimized parameters, which are returned by the DE and Nelder-Mead algorithms, are presented in Table III. Note that the initial values of the parameters in Table III are required by the DE method.

The obtained nonlinear model is verified by comparing the experimental and simulated inductor current $I_{m}$ under two different levels of the excitation voltage $V_{m}$. As can be seen in Fig. 10, the model can fully describe the nonlinear effect occurring in the ferrite core, although some accuracy is lost when increasing the excitation level.

\section{Application of the Dynamic Transformer Model to a Flyback Converter}

Since the two winding models from the SRIVC and LSE methods in Section V-A achieve the same performance, one of these two results can be combined with the core model in Section V-B to form a dynamic transformer model. A direct validation of the dynamic model is to simulate a flyback converter application as a setup shown in Fig. 11. Basically, the simulation can be carried out by any circuit-based simulators which allow
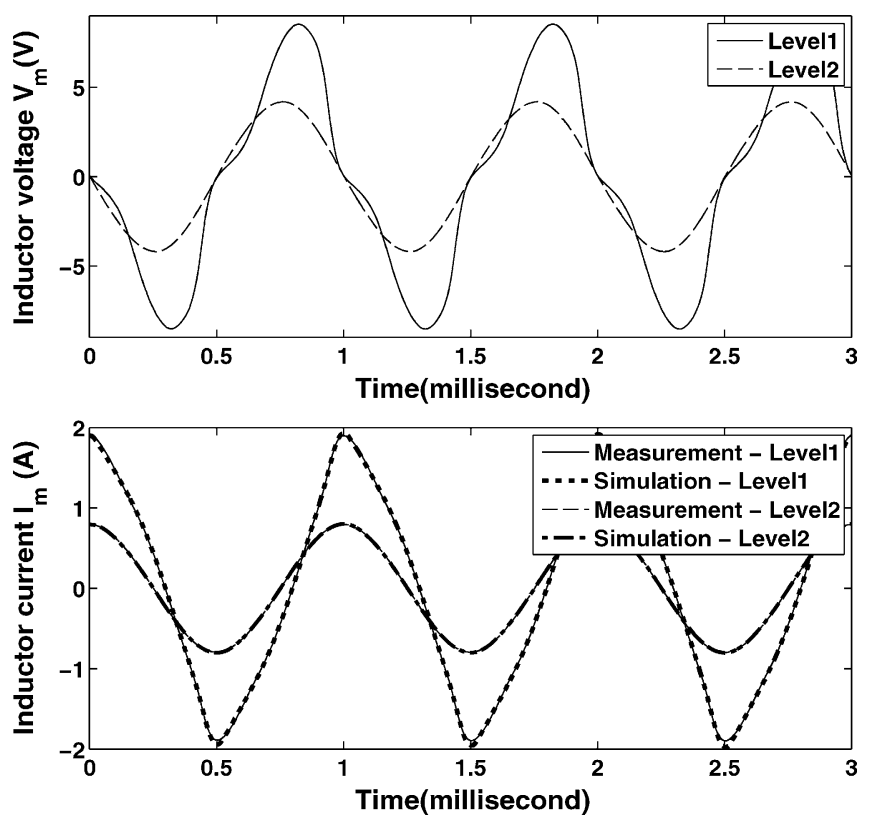

Fig. 10. Nonlinear core model validation under different levels of excitation voltage.

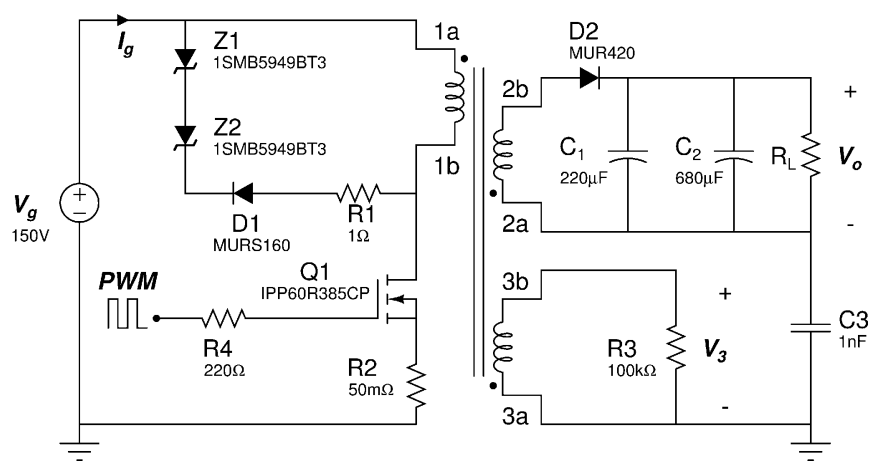

Fig. 11. Circuit prototype of a flyback converter for verification of the dynamic transformer model in Fig. 1.

analog behavioral modeling (ABM), for convenience we go with SimElectronics/Simulink. The ABM capability is required in here for the purpose of the dynamic transformer model implementation. Particularly, the magnetic core $Z_{m}$ in Fig. 1 is achieved by a behavioral block in SimElectronics. Once the block representing $Z_{m}$ is available, the integrated transformer model in Fig. 1 can be easily established.

To fully characterize the transformer behavior, the converter in Fig. 11 should be run within open loop (i.e., with an independent PWM duty cycle). The working condition of the converter is, therefore, decided by the load $R_{s}$, the duty ratio $d$, and the switching frequency $f_{\mathrm{sw}}$ of the PWM signal. Since the transformer model is mainly applied to primary-side sensing control, only the primary current $I_{g}$ and third winding voltage $V_{3}$ are of interest. The transformer is examined under the two working conditions of the flyback converter. Under the first condition, the converter is set up $\left(R_{s}=16.97, d=0.45, f_{\mathrm{sw}}=100 \mathrm{kHz}\right)$ to operate in CCM, while the second working condition is selected $\left(R_{s}=16.829, d=0.38, f_{\mathrm{sw}}=50 \mathrm{kHz}\right)$ to see the 

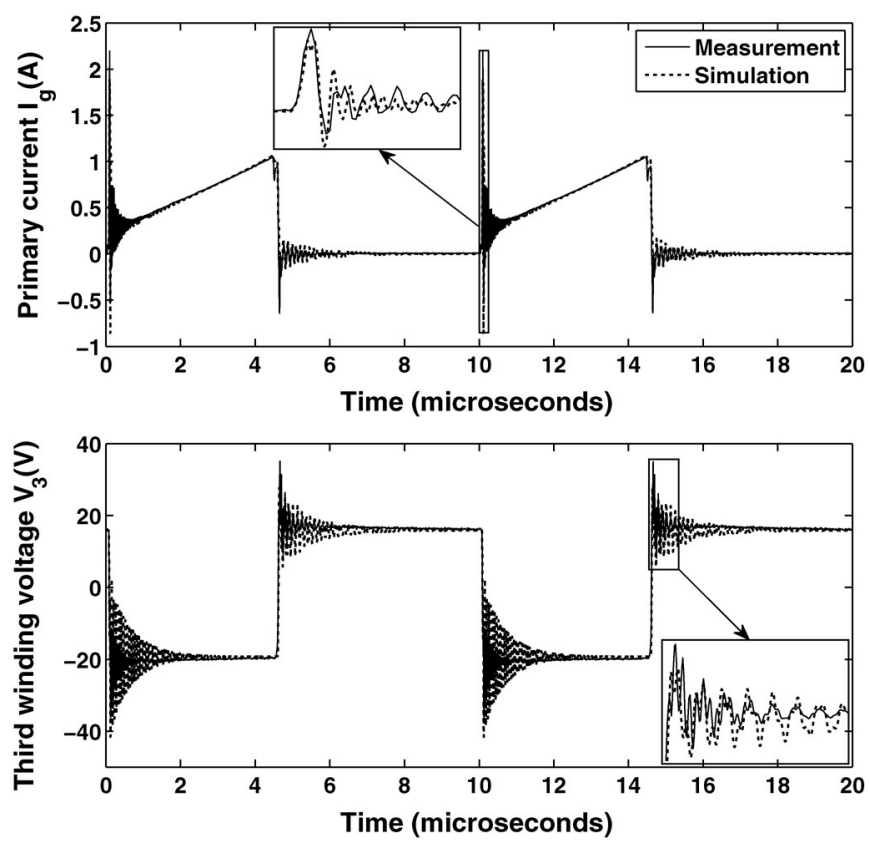

Fig. 12. Comparison between measured and simulated primary current $I_{g}$ and winding voltage $V_{3}$ under $\operatorname{CCM}\left(R_{s}=16.97, d=0.45, f_{\mathrm{sw}}=100 \mathrm{kHz}\right)$.
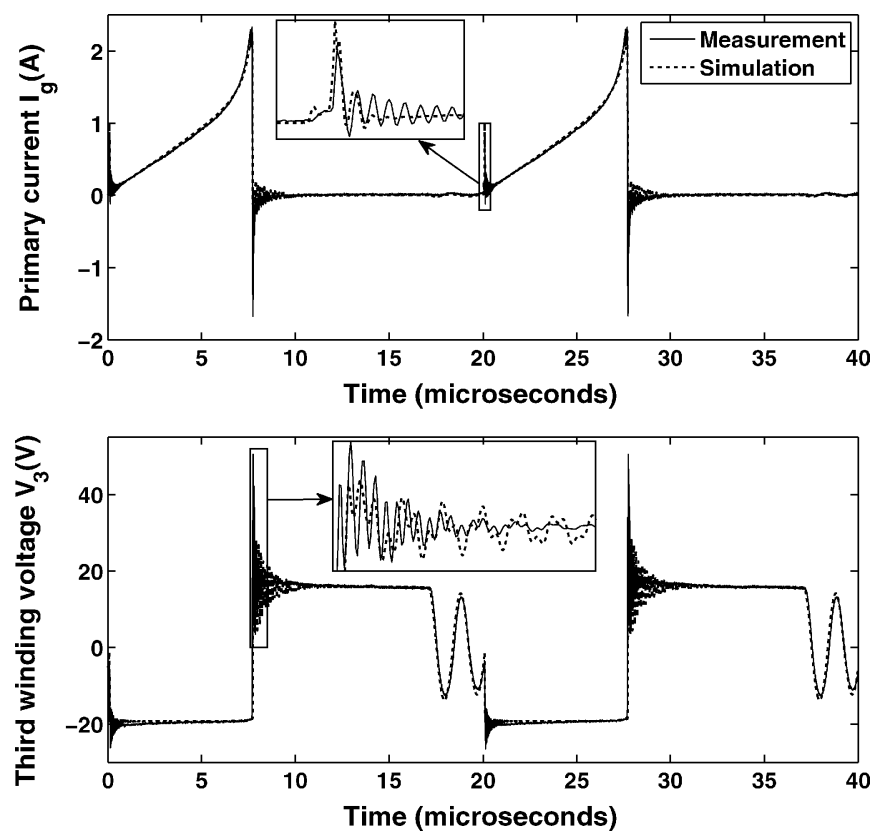

Fig. 13. Comparison between measured and simulated primary current $I_{g}$ and winding voltage $V_{3}$ under DCM $\left(R_{s}=16.829, d=0.38, f_{\mathrm{sw}}=50 \mathrm{kHz}\right)$.

saturation in the core and the ringing effect in DCM. The results for the first and second tests, which are presented in Figs. 12 and 13 , respectively, show very good agreement between the measurement and simulation waveforms. By examining the primary current waveforms in Figs. 12 and 13, specifically during the MOSFET on-time, one can claim that the integrated transformer model can fully represent the behavior of a practical transformer in both linear and nonlinear regions. This conclusion could be further confirmed by observing the ringing in the voltage $V_{3}$ at the end of each switching cycle (see Fig. 13). As can be seen from the magnified windows in Figs. 12 and 13, the simulation is unable to reproduce all the details of the experimental results during the ringing interval after each switching instance. This is due to the imperfection of our simulation program in which the parasitics inside semiconductor devices and between circuit components have been not modeled properly or even ignored. In addition to modeling impairment, the thermal noise which always exists in practice further aggravates the issue.

\section{CONCLUSION}

This study presents a full procedure to extract a nonlinear dynamic model for a 3-winding transformer in time domain. The extraction procedure includes an estimation of the transformer winding model using a time-domain system identification approach and a determination of the core model using an optimization method. The study points out the numerical difficulty, particularly round-off error, associated with the time-domain data collection and proposes using different sensing resistor $R_{s}$ values to improve the estimation results. As demonstrated in the paper, the time-domain approach can provide a winding model at least as accurate as obtained with the frequency response data. With a longer data time series record, a more accurate transformer model is obtained, particularly at low frequencies. Though the selection of different sensing resistor values for different experiments is quite tedious, the time-domain approach requires only simple measurement equipment (say a digital oscilloscope) and most importantly offers an easy way to calculate winding model parameters separately. Since the same transformer is employed to get the data for core model estimation, the obtained model can accurately predict all the nonlinearity occurring throughout the operating regime. In addition, the use of the air-gap length as a variable for estimation, significantly, improves the model accuracy. The results in this paper are valid for both small and large signals and are useful for controller design, system validation purpose, etc.

\section{ACKNOWLEDGMENT}

The authors would like to thank J. Kinsella of NUI Maynooth, Dr. P. Perry of Pilot Photonics, and L. Carroll of University College Dublin for the contributions to this work.

\section{REFERENCES}

[1] P. Hsieh, L. Lee, and K. Hsueh. (2010, Apr.). Driving higher levels of efficiency in lighting through primary side regulation. Fairchild Semiconductor, Taipei, Taiwan. [Online]. Available: http://www.ledinside.com/ knowledge_20100429

[2] A. Dauhajre, "Modeling and estimation of leakage phenomena in magnetic circuit," Ph.D. dissertation, California Inst. Technol., Pasadena, USA, 1986.

[3] A. Keyhani, S. M. Miri, and S. Hao, "Parameter estimation for power transformer models from time-domain data," IEEE Trans. Power Del., vol. 1, no. 3, pp. 140-146, Jul. 1986.

[4] J. Bak-Jenson, B. Bak-Jenson, S. Mikkelsen, and C. Jensen, "Parametric identification in potential transformer modelling," IEEE Trans. Power Del., vol. 7, no. 1, pp. 70-76, Jan. 1992.

[5] V. A. Niemela, H. A. Owen, and W. T. G., "Cross-coupled-secondaries model for multi-winding transformers with parameter values calculated from short-circuit impedance," in Proc. 21 st Annu. IEEE Power Electron. Spec. Conf., San Antonio, TX, USA, Jun. 1990, pp. 822-830. 
[6] J. Pleite, R. Prieto, R. Asensi, J. Cobos, and E. Olias, "Obtaining a frequency-dependent and distributed-effects model of magnetic components from actual measurements," IEEE Trans. Magn., vol. 35, no. 6, pp. 4490-4502, Nov. 1999.

[7] K. Ngo and S. Srinivas, P. Nakmahachalasint, "Broadband extended cantilever model for magnetic component windings," IEEE Trans. Power Electron., vol. 16, no. 4, pp. 551-557, Jul. 2001.

[8] D. Jiles and D. Atherton, "Theory of ferromagnetic hysteresis," J. Magn. Magn. Mater., vol. 61, no. 1/2, pp. 48-60, 1986.

[9] D. Jiles, "Frequency dependence of hysteresis curves in 'non-conducting' magnetic materials," IEEE Trans. Magn., vol. 29, no. 6, pp. 3490-3492, Nov. 1993.

[10] N. Schmidt and H. Guldner, "A simple method to determine dynamic hysteresis loops of soft magnetic materials," IEEE Trans. Magn., vol. 32, no. 2, pp. 489-496, Mar. 1996

[11] P. Wilson and J. Ross, "Definition and application of magnetic material metrics in modeling and optimization," IEEE Trans. Magn., vol. 37, no. 5, pp. 3774-3780, Sep. 2001

[12] P. Nakmahachalasint, K. Ngo, and L. Vu-Quoc, "A static hysteresis model for power ferrites," IEEE Trans. Power Electron., vol. 17, no. 4, pp. $453-$ 460, Jul. 2002.

[13] S. Voss. (2006). Equivalent circuit modelling for wire-wound coils and transformers in power electronics. Ph.D. dissertation, Univ. ErlangenNuremberg, Erlangen, Germany [Online]. Available: http://www.opus.ub. uni-erlangen.de/opus/volltexte/2006/402/

[14] E. Dallago, G. Sassone, and G. Venchi, "High-frequency power transformer model for circuit simulation," IEEE Trans. Power Electron., vol. 12, no. 4, pp. 664-670, Jul. 1997.

[15] R. Prieto, R. Asensi, C. Fernandez, J. Oliver, and J. Cobos, "Bridging the gap between FEA field solution and the magnetic component model," IEEE Trans. Power Electron., vol. 22, no. 3, pp. 943-951, May 2007.

[16] G. Rao and H. Unbehauen, "Identification of continuous-time systems," Proc. IEE Contr. Theor. Appl., vol. 153, no. 2, pp. 185-220, Mar. 2006.

[17] P. Young, H. Garnier, and M. Gilson, "An optimal instrumental variable approach for identifying hybrid continuous-time box-jenkins models," in Proc. 14th IFAC Symp. Syst. Identificat., Newcastle, Australia, Mar. 2006, pp. $225-230$.

[18] H. Garnier, M. Gilson, and V. Laurain. (Nov. 2009). CONTSID toolbox for MATLAB (version 5.0). CRAN, Nancy University, Nancy, France. [Online]. Available: http://www.iris.cran.uhp-nancy.fr/contsid/

[19] L. Ljung, System Identification-Theory For the User, 2nd ed. Upper Saddle River, NJ, USA: Prentice-Hall, 1999.

[20] R. Araujo, A. Leite, and D. Freitas, "Indirect parameter estimation of continuous-time systems using discrete time," in Proc. IEEE 29th Annu. Conf. Ind. Electron. Soc., Nov. 2003, vol. 1, pp. 600-605.

[21] Agilent Technologies. (Jun. 2009). Agilent impedance measurement handbook. [Online]. Available: http://cp.literature.agilent.com/litweb/pdf/ 5950-3000.pdf

[22] K. V. Price, S. R. M. , and J. A. Lampinen, Differential Evolution: A Practical Approach to Global Optimization. New York, NY, USA: SpringerVerlag, 2005

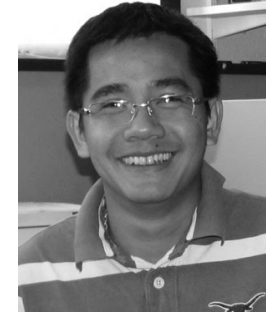

Tue T. Vu (S'13) was born in Binh Duong, Vietnam. He received the Bachelor's degree in electronic engineering (with Hons.) from the Ho Chi Minh City University of Technology, Ho Chi Minh, Vietnam, and the Master's degree in electronic engineering from Dublin City University, Dublin, Ireland, in 2007 and 2010 , respectively. Since 2010, he has been working toward the Ph.D. degree in the Dynamic and Control Research Group, National University of Ireland, Maynooth, Ireland.

His research interests include transformer model identification, controller design, and efficiency optimization for power converters.

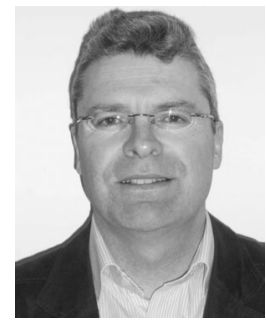

Seamus O'Driscoll received the B.E. (Hons.) and M.E. degrees in electronic engineering from Dublin City University, Dublin, Ireland, in 1986 and 1988, respectively.

He was with Digital Equipment Corporation in the design of Mainframe Computer Power Systems and with Artesyn Ltd. in the design of specialist dc-dc and ac-dc power converter modules for the telecommunications industry. He is currently with Texas Instruments, Cork, Ireland, as a Systems Architect for next-generation digital controller ICs for ac-dc power converters for applications ranging from computer to lighting.

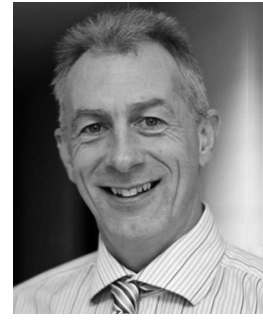

John V. Ringwood (M'87-SM'97) received the Dip. degree in electrical engineering from the Dublin Institute of Technology, Dublin, Ireland, and the Ph.D. degree in control systems from Strathclyde University, Glasgow, U.K., in 1981 and 1985, respectively.

He was with the School of Electronic Engineering, Dublin City University, from 1985 to 2000 and during that time he also holds visiting positions with Massey University and the University of Auckland in New Zealand. He is currently a Professor of electronic engineering with the National University of Ireland, Maynooth, Ireland. His research interests include time series modeling, wave energy systems, modeling, control and fault detection for industrial systems, and biomedical engineering.

Dr. Ringwood is a Chartered Engineer and a Fellow of Engineers Ireland. 